\title{
Predictors of foeto-maternal outcome of patients with abruptio placenta at Bugando Medical Centre, Tanzania
}

\author{
KEVIN NANDONDE ${ }^{1}$, DISMAS MATOVELO ${ }^{1 *}$, ANTHONY MASSINDE ${ }^{1,2}$ and RICHARD RUMANYIKA ${ }^{1,2}$ \\ ${ }^{1}$ Department of Obstetrics and Gynaecology, Catholic University of Health and Allied Sciences P.O. Box 1464, \\ Mwanza, Tanzania \\ ${ }^{2}$ Department of Obstetrics and Gynaecology, Bugando Medical Centre P.O. Box 1370, Mwanza, Tanzania
}

\begin{abstract}
Background: Abruptio placenta (AP) is one of the life-threatening obstetric complications for both the foetus and mother. We conducted this study to determine the incidence and predictors of adverse outcomes of abruptio placenta at Bugando Medical Centre (BMC) in northwest Tanzania.

Methods: This descriptive cross-sectional study was conducted from October 2012 to April 2013. Women with clinical features of abruptio placenta attending BMC were recruited. Data were collected using a structured checklist and analysed with STATA 11.

Results: A total of 3,800 deliveries occurred during study period. The incidence of AP was $2.5 \%$ (95/3800). Among patients with AP, 49 (51.6\%) delivered by Caesarean section. Three (3.2\%) maternal deaths occurred. These deaths were strongly associated with the presence of maternal anaemia $(\mathrm{p}<0.05)$, and postpartum haemorrhage $(\mathrm{p}<0.05)$. The foetal adverse outcomes were prematurity 78 (82.1\%), foetal distress 65 (68.4\%), low birth weight $46(48.4 \%)$ and intrauterine foetal deaths 30 (31.6\%). Perinatal deaths occurred in $52(54.7 \%)$ of the cases, and were predicted by low birth weight $(p<0.001)$, vaginal delivery $(p=0.001)$, birth asphyxia $(p<0.001)$, and retroplacental clot $(>700 \mathrm{ml})$ $(\mathrm{p}<0.001)$.

Conclusion: The incidence of AP at BMC is high and characterised by poor maternal and foetal outcomes. Patients with AP should be delivered promptly to improve their survival.
\end{abstract}

Keywords: abruptio placenta, maternal, foetal, outcomes, Tanzania

\section{Introduction}

Abruptio placenta (AP) is a grave obstetrical emergency that refers to bleeding at the decidualplacental interface, leading to placental detachment from 28 weeks of gestational age and prior to delivery of the foetus. It deprives the foetus of oxygen and nutrition, leading to both short term and long-term consequences among survivors (Rasmussen et al., 2000; Oyelese \& Ananth, 2006). It is among the leading causes of maternal and perinatal mortality and morbidity worldwide (Witlin, 2001; Tikkanen, 2010). The World Health Organization estimates showed maternal mortality rates due to AP worldwide was $2.1 \%$ and foetal perinatal mortality rate was $15 \%$ while its incidence was $0.65 \%$ (Neilson, 2011).

In developed countries, approximately $10 \%$ of all preterm births and $10-20 \%$ of all perinatal deaths are due to abruptio placenta (Tikkanen, 2011). In developing countries, more maternal deaths are believed to be caused by haemorrhage (Neilson, 2003; Oyelese \& Ananth, 2006). In Tanzania abruptio placenta accounts for $2.6 \%$ of maternal and foetal mortality, despite the high rate (96\%) of mothers attending antenatal care (TDHS, 2010). At the Muhimbili National Hospital, Muhimbili, AP has been associated with about a quarter of all stillbirths and neonatal deaths (Kidanto et al., 2009).

Despite the high prevalence of maternal and foetal death due to abruptio placenta, there is limited information about the condition in north-western Tanzania. This study was therefore, conducted to determine the incidence and predictors of adverse outcomes of abruptio placenta at Bugando Medical Centre (BMC) in northwest Tanzania.

\section{Materials and Methods}

\footnotetext{
*Correspondence: E-mail: magonza77@yahoo.co.uk
} 


\section{Study site}

A cross-sectional study was conducted from October 2012 to April 2013 at Bugando Medical Centre (BMC) in Mwanza, Tanzania. The centre serves both as a referral consultant and a University teaching hospital. The hospital annual average number of deliveries is 6,825 (BMC unpublished reports).

\section{Study subjects}

All pregnant women with features of abruptio placenta from 28 weeks of gestation were included in the study. Delivery outside BMC was an exclusion criterion. Clinical diagnosis of abruptio placenta was reached in presence of one or more of the following characteristics: painful vaginal bleeding accompanied by hypertonus uterine contractions, tender uterus \pm nonreassuring foetal heart rate/ foetal distress, foetal demise, pallor and rapid breathing with hypotension (systolic Blood Pressure < 90mmHg) (Elsasser et al., 2010). The presence of retroplacental clots post-delivery supported the final diagnosis (Elsasser et al., 2010). Thus participants with AP and their babies meeting who met the inclusion criteria were cordially invited to participate in the study. Data was collected serially until the sample size was reached. Sample size was determined by a formula described by Yamane (1967) considering the number of AP patients at BMC from previous year was 49 and level of precision or sampling of error which is $\pm 5 \%$.

Participants' information was extracted using structured checklist. Apart from thorough history taking and physical examinations, bedside obstetric ultrasound and examination under anaesthesia were incorporated for a few selected cases to exclude placenta praevia, posterior placenta and other causes of antepartum haemorrhage (APH). Each participant gestational age was calculated using Naegele's rule (Baskett, 2000). Systolic blood pressure was measured in $\mathrm{mmHg}$ using a manual sphygmomanometer and foetal heart rate counted for one minute using a pinard stethoscope. Participants were followed up for the mode of delivery reached. Retroplacental clots were measured by estimating the volume in a kidney dish able to hold $500 \mathrm{~mL}$ of fluid. The Apgar score and weight of the baby were measured accordingly. Mothers and their babies were followed for a period of 7 days post-delivery; those who were discharged home before seven days were asked to attend the postnatal care clinic on day 7. In addition, patients' phone numbers were taken for tracing and home visits when necessary.

\section{Laboratory analysis}

Venepuncture was done for all participating patients to determine bedside clotting time, platelet counts, haemoglobin level, prothrombin time and serum creatinine level. A normal blood clotting time was considered to be 5 to 11 minutes; normal platelet count was considered to be $150-400 \mathrm{x}$ $10^{9} / \mathrm{L}$ while the normal range of haemoglobin and prothrombin time (PT) was considered to be 11$16 \mathrm{~g} / \mathrm{dl}$ and 10-14 seconds, respectively (Kratz et al., 2004). The normal range for serum creatinine was $65-139 \mathrm{mmol} / \mathrm{L}$. All laboratory values, clinical evaluations and management outcomes of patient were documented in the case record forms. Predictors of maternal outcome included clinical presentations (systolic blood pressure, high pulse rate, vaginal bleeding and signs of disseminated intravascular coagulopathy (DIC)), laboratory results (bedside clotting time, serum creatinine level, haemoglobin level, prothrombin time and platelet level), size of retroplacental clot and management received (mode of delivery, number of blood transfusion units and fresh frozen plasma (FFP), intensive care unit (ICU) admissions (only patient with hypovolemic shock, DIC and acute renal failure (persisted oliguria i.e., urine output $>500 \mathrm{mls} / 24 \mathrm{hrs}$ or presence of elevated serum creatinine $\geq 140 \mathrm{mmol} / \mathrm{l}$ ), and peripartum hysterectomy.

Predictors for foetal outcome were gestation age, prematurity, birth weight, Apgar score, foetal heart rate status, mode of delivery and size of retro-placental clot. Prolonged hospital stay was defined as either staying in hospital for more than 24 hours if delivered 
vaginally or more than 3 days if delivered by Caesarean section (C-section). The tool was tested before the beginning of the actual data collection.

\section{Data analysis}

Data was entered into a computer in Epi-info Data version 3.1 software. Double entry of data was done to ensure correctness of information. Data analysis was done using STATA 11 (College Station, Texas, USA). A t-test statistic was used to compare the means of continuous data. Categorical variables were summarized as proportions and analysed by Chi-square or Fisher's exact test. $\mathrm{P}$-values of less than 0.05 were considered significant.

\section{Ethical consideration}

Ethical clearance was provided by the joint Ethical Committee of Catholic University of Health and Allied Sciences and BMC. Permission to conduct the study was sought from the BMC Department of Obstetrics and Gynaecology. Strict ethical standards were upheld throughout the study to ensure privacy and confidentiality. Registration and identification numbers were used rather than names or other unique identifiers. Patients were informed of the nature of the study and consent was obtained from each respondent. All patients were managed according to the hospital protocol.

\section{Results}

A total of 3,797 cases were admitted during the study period. Ninety-five (2.5\%) patients had abruptio placenta (AP). The mean age of study participants was $28.04 \pm 6.03$ years. About 76 $(80 \%)$ of participants were multipara, most of them (70.5\%) were married and $36.8 \%$ were housewives (Table 1).

Table 1: Baseline characteristics of study participants

\begin{tabular}{|c|c|c|c|}
\hline Characteristics & Response & Frequency & Percentage \\
\hline \multirow[t]{3}{*}{ Maternal age (years) } & $\leq 25$ & 44 & 46.3 \\
\hline & $26-35$ & 34 & 35.8 \\
\hline & $36-45$ & 17 & 17.9 \\
\hline \multirow[t]{4}{*}{ Marital status } & Single & 2 & 2.1 \\
\hline & Married & 67 & 70.5 \\
\hline & Cohabiting & 23 & 24.2 \\
\hline & Separated & 3 & 3.2 \\
\hline \multirow[t]{5}{*}{ Occupation } & Housewife & 35 & 36.8 \\
\hline & Student & 9 & 9.5 \\
\hline & Employee & 15 & 15.8 \\
\hline & Business & 26 & 27.4 \\
\hline & Peasant & 10 & 10.5 \\
\hline \multirow[t]{4}{*}{ Education level } & No formal education & 6 & 6.3 \\
\hline & Primary school & 35 & 36.8 \\
\hline & Secondary school & 41 & 43.2 \\
\hline & College/University & 13 & 13.7 \\
\hline \multirow[t]{3}{*}{ Parity } & Primiparity & 19 & 20.0 \\
\hline & Multiparity (1-4) & 50 & 52.6 \\
\hline & Grand multiparity $(>4)$ & 26 & 27.4 \\
\hline \multirow[t]{3}{*}{ Gestation age (weeks) } & $28-32$ & 44 & 46.3 \\
\hline & $33-36$ & 34 & 35.8 \\
\hline & $37-45$ & 17 & 17.9 \\
\hline \multirow[t]{3}{*}{ Haemoglobin level (gm/dl) } & $\geq 11.0$ & 6 & 6.3 \\
\hline & $6-10$ & 63 & 66.3 \\
\hline & $<6$ & 26 & 27.4 \\
\hline \multirow[t]{2}{*}{ Mode of delivery* } & Vaginal & 45 & 47.4 \\
\hline & Caesarean & 49 & 51.6 \\
\hline
\end{tabular}

* One pregnant woman with AP died prior to delivery 
Maternal mortality in this study was $3(3.2 \%)$. The majority of participants $82(86.3 \%)$ had prolonged hospital stay due to complications that arose during the course of illness. On admission 30 women had no foetal heart activity, 23 of them delivered vaginally, six of them delivered by C-section because of life-threatening haemorrhage and one woman died before delivery. Sixty five women had foetal heart activity on admission, 22 of them delivered vaginally and 43 delivered by C-section. Approximately $60 \%$ of those with foetal heart activity on admission underwent emergency $C$-section which showed to reduce perinatal death $\left(\chi^{2}=18.6, p<0.001\right)$. Out of 95 patients, $69.5 \%$ needed at least one blood product transfusion either or both due to hypovolemic shock and DIC. Other maternal complications observed during study period were anaemia in $89(93.7 \%)$, coagulopathy in $62(65.3 \%)$, hysterectomy in $2(2.1 \%)$, acute renal failure in 20 (21.6\%), primary postpartum haemorrhage in 35 (36.8\%), hypovolemic shock in $15(15.8 \%)$ and a need for intensive care in 13 (13.7\%) women (Table 2 ).

Almost all participants who were diagnosed with acute renal injury $20(21.6 \%)$ post delivery following AP recovered with infusion of crystalloid and none required dialysis. Perinatal death in this study was 52 (54.7\%). About 30(34.7\%) were fresh stillbirths, whereas 25 stillbirth were confirmed intrauterine foetal deaths (IUFD) on admission and 5 foetuses died during the course of labour. The remaining 22 babies (23.2\%) were born alive but died in the $1^{\text {st }}$ week of life (neonatal period) due to severe birth asphyxia $(15,60 \%)$ or prematurity-related complications ( 36 , $78 \%)$. Of these perinatal deaths, 35 (75\%) were delivered vaginally and 17 (35\%) by C-section (Table 2).

Table 2: Maternal and Foetal adverse outcomes

\begin{tabular}{llll}
\hline Outcome & Variable & Frequency & Percentages \\
\hline Maternal outcome & Prolonged hospital stay & 82 & 86.3 \\
& Maternal death & 3 & 3.2 \\
& Acute kidney injury & 20 & 21.6 \\
& ICU admission & 13 & 13.7 \\
& Hysterectomy & 2 & 2.1 \\
& Need for blood transfusion & 66 & 69.5 \\
& Anaemia & 89 & 93.7 \\
DIC/ Coagulopathy & 62 & 65.3 \\
Foetal outcome & PPH & 26 & 27.4 \\
& Shock & 15 & 15.8 \\
& Foetal distress & 20 & 21.1 \\
Low Apgar score (below 7) & 24 & 25.3 \\
& Low birth weight & 46 & 48.4 \\
& Stillbirth & 30 & 34.7 \\
& Early neonatal death & 22 & 23.2 \\
\hline
\end{tabular}

Predictors that were associated with maternal death were maternal anaemia on admission and primary postpartum haemorrhage $(p=0.021)$. Admission to ICU increased the likelihood of survival in participants with acute renal failure (ARF), hypovolemic shock and DIC $(p=0.043)$. However, there was no significant association between maternal deaths and age $(p=0.125)$, blood transfusion $(p=0.310)$, shock $(p=0.406)$ or prolonged bedside clotting time $(p=0.273)$ (Table 3$)$. 
Table 3: Predictors of maternal death and survival

\begin{tabular}{|c|c|c|c|c|}
\hline \multicolumn{2}{|c|}{ Patients characteristics } & \multicolumn{2}{|c|}{ Maternal death } & \multirow{2}{*}{ P-value* } \\
\hline & & Yes; N (\%) & No; N (\%) & \\
\hline \multirow[t]{3}{*}{ Age } & $<25$ & $0(0.0)$ & $44(100.0)$ & \\
\hline & $25-35$ & $3(8.8)$ & $31(91.2)$ & 0.125 \\
\hline & $>35$ & $0(0.0)$ & $17(100.0)$ & \\
\hline \multirow[t]{2}{*}{ DIC } & Yes & $3(4.8)$ & $59(95.2)$ & \\
\hline & No & $0(0.0)$ & $33(1.0)$ & 0.273 \\
\hline \multirow[t]{3}{*}{ Anaemia } & $\geq 11$ & 1(16.7) & $5(83.3)$ & \\
\hline & $6-10$ & $0(0.0)$ & $66(100.0)$ & 0.021 \\
\hline & $<5$ & $2(8.7)$ & $21(91.3)$ & \\
\hline \multirow[t]{3}{*}{ Blood transfusion } & None & $1(3.6)$ & $27(96.40)$ & \\
\hline & $1-3$ & $1(1.8)$ & $56(98.2)$ & 0.310 \\
\hline & $\geq 4$ & $1(10.0)$ & $9(90.0)$ & \\
\hline \multirow[t]{3}{*}{ FFB } & None & $2(0.03)$ & $65(97)$ & \\
\hline & $1-3$ units & $1(0.04)$ & $26(96.3)$ & 0.310 \\
\hline & $\geq 4$ & $O(0.0)$ & $1(1.0)$ & \\
\hline \multirow[t]{2}{*}{ Shock } & Yes & $1(6.67)$ & $14(93 \cdot 3)$ & \\
\hline & No & $2(2.50)$ & $78(97 \cdot 50)$ & 0.400 \\
\hline \multirow[t]{2}{*}{ ICU admission } & Yes & $2(15 \cdot 38)$ & $11(84.62)$ & \\
\hline & No & $1(1.22)$ & $81(98.78)$ & 0.043 \\
\hline \multirow[t]{2}{*}{ PPH } & Yes & $2(7.69)$ & $24(92.31)$ & \\
\hline & No & $0(0.00)$ & $68(100)$ & 0.002 \\
\hline \multirow[t]{2}{*}{ Clotting time(min) } & $<11$ & $0(0.00)$ & $33(100)$ & \\
\hline & $>12$ & $3(3.16)$ & $59(95.16)$ & $0.2 / 3$ \\
\hline
\end{tabular}

*All were analysed by Fisher's exact test

Predictors that were associated with prolonged hospital stay were shock $(p=0.045)$, vaginal delivery $(p<0.001)$ and admission into ICU $(p=0.0015)$. On the other hand, caesarean delivery ( $p$ $<0.001)$ and amniotomy combined with oxytocin $(p<0.001)$ were found to shorten the duration of hospital stay. The postpartum haemorrhage $(p=0.381)$, maternal anaemia $(p=0.155)$, hysterectomy $(p=0.916)$ and acute kidney failure $(p=0.218)$ were not associated with the duration of hospital stay (Table 4).

Table 4: Predictors of prolonged hospital stay

\begin{tabular}{|c|c|c|c|c|}
\hline \multicolumn{2}{|c|}{ Patient characteristics } & \multicolumn{2}{|c|}{ Prolonged hospital stay } & \multirow[t]{2}{*}{ P-value } \\
\hline & & Yes; N (\%) & No; N (\%) & \\
\hline \multirow[t]{2}{*}{$\mathrm{PPH}$} & Yes & $10(38.46)$ & $16(61.54)$ & $0.381 * *$ \\
\hline & No & $35(51.47)$ & $33(48.53)$ & \\
\hline \multirow[t]{2}{*}{ Mode of delivery } & SVD & $41(91.0)$ & $4(8.90)$ & $<0.001 *$ \\
\hline & C-Section & $10(20.4)$ & $39(79.6)$ & \\
\hline \multirow[t]{2}{*}{ Shock } & Yes & $9(60.0)$ & $6(40.0)$ & $0.0543 * *$ \\
\hline & No & $42(52.5)$ & $38(47.5)$ & \\
\hline \multirow[t]{2}{*}{ ICU admission } & Yes & $11(84.62)$ & $2(15.38)$ & $0.015^{*}$ \\
\hline & No & $40(48.78)$ & $42(51.22)$ & \\
\hline \multirow[t]{3}{*}{ Anaemia (mg/dl) } & $\geq 11$ & $2(33.33)$ & $4(66.67)$ & \\
\hline & $6-10$ & $33(50.0)$ & $33(50.0)$ & $0.155^{*}$ \\
\hline & $<6$ & $16(69.57)$ & $7(30.43)$ & \\
\hline \multirow[t]{2}{*}{ Hysterectomy } & Yes & $1(50.0)$ & $1(50.0)$ & \\
\hline & No & $50(53.76)$ & $43(46.24)$ & $0.916^{*}$ \\
\hline
\end{tabular}

Predictors that were strongly associated with perinatal death were low birth weight $(p<0.001)$, foetal heart rate on admission ( $p<0.001)$, maternal anaemia $(p=0.003)$, vaginal delivery $(p=0.001)$, low Apgar score $(p<0.001)$ and size of retroplacental clot $(p<0.001)$. Caesarean section has shown to reduce perinatal death $(p=0.001)$. However, there was no association between perinatal death with maternal age $(p=0.085)$, gestational age $(p=0.403)$, or sex of the foetus $(p=0.422)$ (Table 5$)$. 
Table 5: Predictors of perinatal deaths

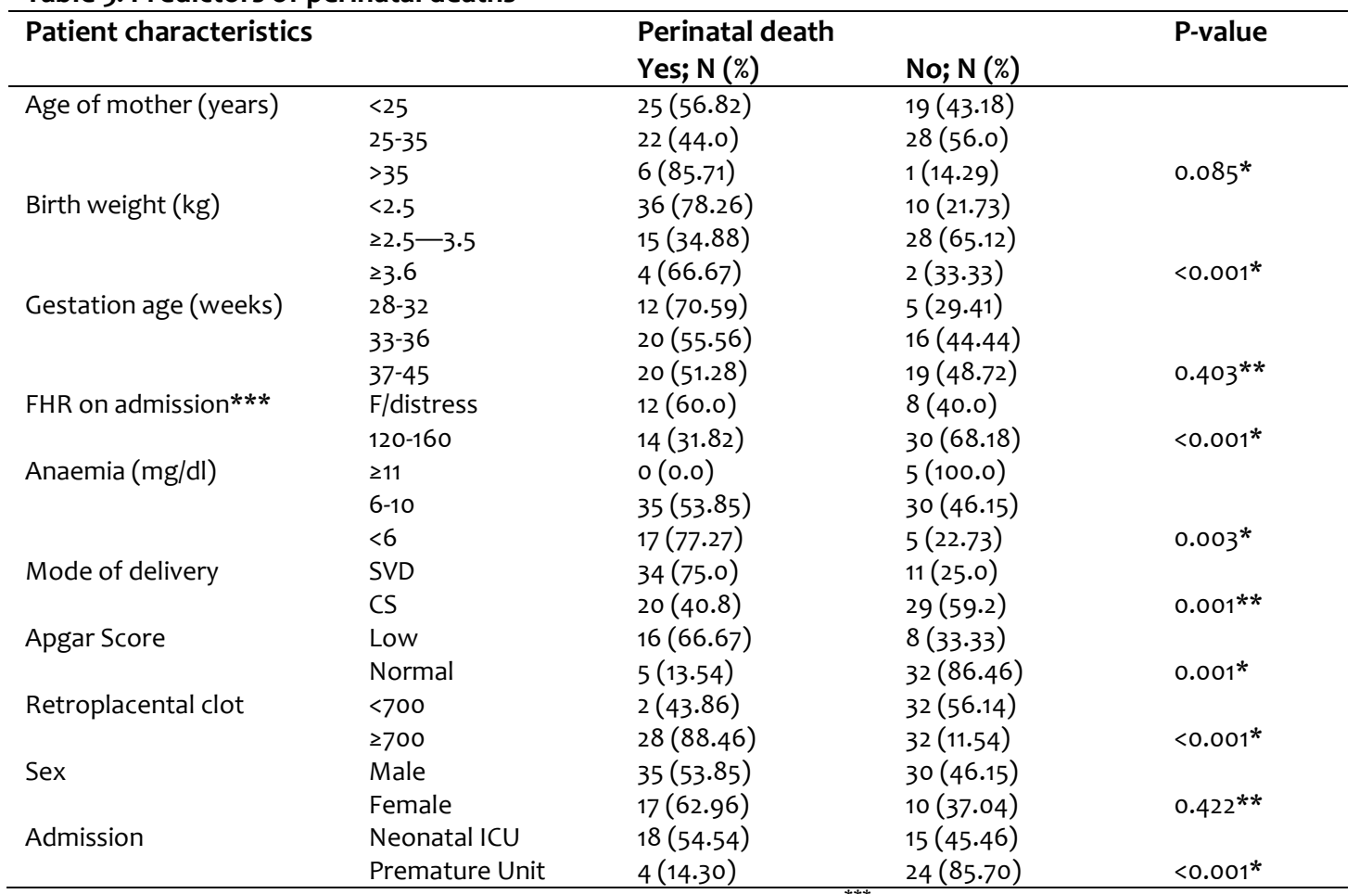

*Analysed by Fisher's exact test; **Analysed by Pearson chi-square test; ${ }^{* * *}$ Thirty women with AP had intrauterine foetal death on admission.

\section{Discussion}

This study has demonstrated a clear higher risk for maternal and neonatal adverse outcomes among women with abruptio placenta. The incidence of abruptio placenta in this study was low and comparable to other studies in developing countries (Wandabwa et al., 2007; Hossain et al., 2010; Mutihir \& Eka, 2011). However, studies done in developed countries reported a much lower incidence (Oyelese \& Ananth 2006; Tikkanen, 2011). Early detection and management of modifiable risk factors for placenta abruption such as hypertension and diabetes in developed countries may have helped to reduce the incidence of abruptio placenta (Sheiner et al., 2003; Dafallah \& Babikir, 2004; Hung et al., 2007). In addition, abruptio placenta is more common among blacks than whites owing to genetic predisposition among blacks (Shen et al., 2008).

Maternal adverse outcomes such as anaemia, hysterectomy, acute renal failure, primary postpartum haemorrhage, hypovolemic shock and maternal deaths found in this study have been reported elsewhere in developing countries (Ozumba, 1989; Prual et al., 2000). Although similar findings have been reported by other studies, we cannot conclude that anaemia is a direct adverse outcome of abruptio placenta as most patients presented without antenatal records. Also, since antepartum haemorrhage causes acute haemorrhage, the haemoglobin level does not necessarily reflect the severity of anaemia. The high frequency of maternal anaemia could be explained by underlying chronic maternal nutritional deficits common in the Lake Victoria zone of Tanzania (TDHS, 2010).

Prolonged clotting time was found in about two thirds of the mothers. The clotting time was longer than those reported from other studies (Abbasi et al., 2008; Bibir et al., 2009; Jabeen \& Gul, 2011). The reason for the higher event of DIC in this study may be attributed to late referrals from lower health facilities. However mortality owing to DIC in this study was lower and similar to findings from other studies (Abbasi et al., 2008; Bibi et al., 2009; Jabeen \& Gul, 2011). 
This may be attributed by the availability of life saving blood products such as fresh frozen plasma, platelet concentrates and fresh blood during resuscitation. Blood transfusion was needed in majority of cases. Several studies have shown blood transfusion to be required in over half of cases (Abbasi et al., 2008; Bibi et al., 2009; Ozumba, 1989).

The event of PPH in AP was higher than findings from other studies elsewhere (Ozumba, 1989; Sarwar et al. 2006; Abbasi et al. 2008; Bibi et al. 2009; Jabeen \& Gul 2011). Such a higher incidence is likely attributable to cases with coagulopathy.

Caesarean deliveries were significantly associated with reduced perinatal death. This is in contrast to other studies in which vaginal delivery was the commonest, with C-sections done in few cases. In other studies, most foetuses have been stable and hence afforded the time for artificial rupture of membrane and augmentation of labour (Ozumba, 1989; Hossain et al. 2010). The higher incidence of C-section in our setup is likely to be attributable to late presentation to the hospital with compromised foetal status, hence requiring an urgent delivery.

The low rate of hysterectomy because of primary PPH in our study was comparable to a study in Abbottabad (Sarwar et al. 2006). Similarly, the rate of maternal shock was comparable to findings by Pitaphorm \& Sukcharoen (2006). Adult intensive care unit admission was necessary in about one-tenth the cases. Other studies have also reported a similar low rate of ICU admission (Bibi et al., 2009; Mbaga et al., 2011). Late presentation to the hospital with other co-morbidities necessitated close monitoring in ICU in our setting. In this study, like in other previous studies, ICU admission was significantly associated with reduced maternal death (Neilson, 2003; Pitaphrom \& Sukcharoen 2006; Tikkanen, 2011).

The low maternal mortality in this study was comparable with other studies from developing countries (Dafallah \& Babikir 2004; Pitaphrom \& Sukcharoen 2006; Sarwar et al., 2006; Hossain et al., 2010). In contrast, studies from developed world have reported lower mortality due to early referral to the hospital, leading to less adverse outcomes such as DIC and availability of fractionated clotting factors and blood products for resuscitation that improved maternal survival in developed world (Ananth et al., 1999; Oyelese \& Ananth 2006; Abbasi et al., 2008; Tikkanen, 2011).

Prolonged hospital stay was found in the majority of cases; and it was associated with maternal shock and ICU admission. In other studies prolonged hospital stay ranged from $28 \%$ to 47\%. The difference is associated with varying definition of normal hospital stay in different hospitals to patient who had caesarean section and vaginal delivery (Sheiner et al., 2003; Tikkanen, 2011).

Foetal adverse outcomes were perinatal deaths, prematurity, low birth weight, and birth asphyxia. It may be argued that most of neonates who lost their lives were potentially salvageable. The higher perinatal deaths were comparable to previous studies in the developing world (Naeye et al., 1979; Kramer et al., 1997; Pitaphrom \& Sukcharoen, 2006). Relatively lower perinatal deaths have been reported in developed world (Rasmussen et al., 1996; Ananth et al., 2006; Oyelese \& Ananth, 2006; Aliyu et al., 2012). The difference is attributable to lack of comprehensive advanced care of premature babies in our setup.

In this study neonatal admission to the intensive care unit (NICU) had high rates of perinatal death. Similar findings have been reported by Hossain et al. (2010) in which admission to the premature unit was protective. This is because most of the neonates who were admitted to NICU had various complications particularly birth asphyxia.

Low birth weight was slightly higher in this study and other developing countries (Ananth et al., 1999; Kayani et al., 2003; Oyelese \& Ananth 2006; Tikkanen et al., 2006; Tikkanen, 2011) due to the fact that they are multifactorial in origin. In this study low birth weight showed a direct link to premature deliveries. In this study there was a lower incidence of AP in mothers with female babies than with male babies, but there was no sex difference in foetal survival observed among the offspring. Sex differences associated with abruptio placenta have been reported by previous studies (Witlin \& Sibai, 2001; Tikkanen et al., 2006). 
Generally, perinatal mortality has been strongly associated with complete AP across the world. In our study, the association was much stronger with low birth weight, birth asphyxia, maternal anaemia, preterm gestation (28-32 weeks), spontaneous vertex delivery as a mode of delivery and high volume of retroplacental clot. A similar finding had been reported by Ananth et al. (1999), where association was stronger with preterm gestation, low birth weight and increased volume of retro-placental clot.

The incidence of AP at BMC is significantly higher with poor foeto-maternal outcomes. The predictors of maternal adverse outcomes were anaemia, PPH and maternal shock, while predictors for perinatal death were low birth weight, birth asphyxia, low Apgar score, retroplacental clot volume above $700 \mathrm{ml}$ and spontaneous vertex delivery. Predictors of adverse foeto-maternal outcomes should be closely monitored with prompt delivery as well as having adequate maternal-neonatal intensive care facilities to improve chance of survival in these vulnerable mothers and babies.

\section{Competing Interest}

The authors declare that they have no competing interests.

\section{Author's contributions}

$\mathrm{KN}$ : involved in the study design, data collection, analysis and interpretation. DM: involved in study design, data collection, analysis and drafted the first manuscript. AM: involved in study design, data collection, analysis and editing the manuscript. RR: involved in study design, data collection, analysis. All authors read, commented on and approved the final version of the manuscript

\section{Acknowledgements}

We are very grateful to all midwives at the maternity unit for their tireless efforts endured in providing maternal and newborn resuscitation of all cases that were recruited in this study. We are also indebted to all women who kindly agreed to participate in the study; without them the study couldn't have been conducted. Dr Benson Kidenya is thanked for his technical support during data analysis.

\section{References}

Abbasi, R. M., Rizwan N., Mumtaz F. \& Farooq S. (2008) Feto maternal outcome among Abruptio placentae cases at a University hospital of Sindh. Journal of Liaquat University of Medical and Health Sciences 7, 106-109.

Aliyu, M.H., Salihu H.M., Lynch, O.N., Alio, A.P. \& Marty, P.J. (2012) Placental abruption, offspring sex, and birth outcomes in a large cohort of mothers. Journal of Maternal-Fetal and Neonatal Medicine 25, 248-252.

Ananth, C.V., Berkowitz G.S., Savitz, D.A. \& Lapinski, R.H. (1999) Placental abruption and adverse perinatal outcomes. JAMA 282, 1646-1651.

Ananth, C.V., Oyelese, Y., Ananth, C.V., Prasad, V., Getahun, D. \& Smulian, J.C. (2006) Evidence of placental abruption as a chronic process: associations with vaginal bleeding early in pregnancy and placental lesions. European Journal of Obstetrics \& Gynecology and Reproductive Biology 128, 15-21.

Bibi, S., Ghaffar S., Pir, M. A. \& Yousfani, S. (2009) Risk factors and clinical outcome of placental abruption: a retrospective analysis. Journal of Pakistan Medical Association 59, 672-674. 
Dafallah, S.E. \& Babikir, H.E. (2004) Risk factors predisposing to abruptio placentae." Maternal and fetal outcome. Saudi Medical Journal 25, 1237-1240.

Elsasser, D.A., Ananth, C.V., Prasad, V., Vintzileos, A.M. \& New Jersey-Placental Abruption Study Investigators. (2010) Diagnosis of placental abruption: relationship between clinical and histopathological findings. European Journal of Obstetrics \& Gynecology and Reproductive Biology 148, 125-130.

Hossain, N., Khan N., Sultana, S.S. \& Khan, N. (2010) Abruptio placenta and adverse pregnancy outcome. Journal of Pakistan Medical Association 60, 443-446.

Hung, T.-H., Hsieh, C., Hsu, J.J., Lo, L.M. \& Chiu, T.H. (2007) Risk factors for placental abruption in an Asian population. Reproductive Sciences 14, 59-65.

Jabeen, M. \& Gul F. (2011) Abruptio placentae: risk factors and perinatal outcome. Journal of Postgraduate Medical Institute (Peshawar-Pakistan) 18, 669-676.

Kayani, S. I., Walkinshaw S., \& Preston, C. (2003) Pregnancy outcome in severe placental abruption. BJOG: An International Journal of Obstetrics \& Gynaecology 110, 679-683.

Kidanto, H.L., Mogren, I., van Roosmalen, J., Thomas, A.N., Massawe, S.N., Nystrom, L. \& Lindmark, G. (2009) Introduction of a qualitative perinatal audit at Muhimbili National Hospital, Dar es Salaam, Tanzania. BMC Pregnancy and Childbirth 9, 45.

Kramer, M.S., Usher, R.H., Pollack, R., Boyd, M. \& Usher, S. (1997) Etilogic determinants of abruptio placentae. Obstetrics \& Gynecology 89, 221-226.

Kratz, A., Ferraro, M., Sluss, P.M. \& Lewandrowski, K.B. (2004) Laboratory reference values. New England Journal of Medicine 351, 1548-1564.

Mbaga, B., Lie, R., Kibiki, G.S., Olomi, R., Kvåle, G. \& Daltveit, A.K. (2011) Transfer of newborns to neonatal care unit: a registry based study in northern Tanzania. BMC Pregnancy and Childbirth 11, 68.

Mutihir, J.T. \& Eka, P.O. (2011) Stillbirths at the Jos University Teaching Hospital: Incidence, risk, and etiological factors. Nigerian Journal of Clinical Practice 14, 14-18.

Naeye, R.L., Tafari, N. \& Marboe, C.C. (1979) Perinatal death due to abruptio placentae in an African city. Acta Obstetricia et Gynecologica Scandinavica 58, 37-40.

Neilson, J.P. (2011) Interventions for treating placental abruption (Review). Health.

Oyelese, Y. \& Ananth, C.V. (2006) Placental abruption. Obstetrics \& Gynecology 108, 1005-1016.

Ozumba, B.C. (1989) Abruptio placentae at The University of Nigeria Teaching Hospital, Enugu: a 3-year study. Australian and New Zealand Journal of Obstetrics and Gynaecology 29, 117-120.

Pitaphrom, A. \& N. Sukcharoen (2006) Pregnancy outcomes in placental abruption. Journal of Medical Association of Thailand 89,1572-1578.

Prual, A., Bouvier-Colle, M. H., Bernis, L. D. \& Breart, G. (2000) Severe maternal morbidity from direct obstetric causes in West Africa: incidence and case fatality rates. Bulletin of the World Health Organization 78, 593-602.

Rasmussen, S., Irgens, L.M., Bergsjø, P. \& Dalaker, K. (1996) The occurrence of placental abruption in Norway 1967-1991. Acta Obstetricia et Gynecologica Scandinavica 75, 222-228.

Rasmussen, S., Irgens, L.M., Bergsjø, P. \& Dalaker, K. (2000) Outcome of pregnancies subsequent to placental abruption: a risk assessment. Acta Obstetricia et Gynecologica Scandinavica 79, 496-501.

Sarwar, I., Abbasi, A.N. \& Islam, A. (2006) Abruptio placentae and its complications at Ayub Teaching Hospital Abbottabad. Journal of Ayub Medical College, Abbottabad 18, 27.

Sheiner, E., Shoham-Vardi, I., Hallak, M., Hadar, A., Gortzak-Uzan, L., Katz, M. \& Mazor, M. (2003). Placental abruption in term pregnancies: clinical significance and obstetric risk factors. Journal of Maternal-Fetal and Neonatal Medicine 13, 45-49.

Shen, T.T., DeFranco, E.A., Stamilio, D.M., Chang, J.J. \& Muglia, L.J. (2008) A population-based study of race-specific risk for placental abruption. BMC Pregnancy and Childbirth 8, 43.

TDHS (2010) Tanzania Demographic and Health Survey 2010. National Bureau of Statistics, Dar es Salaam, Tanzania, ICF Macro Calverton, Maryland, USA, Report; April 2011. 
Tikkanen, M. (2011) Placental abruption: epidemiology, risk factors and consequences. Acta Obstetricia et Gynecologica Scandinavica 90, 140-149.

Tikkanen, M., Nuutila, M., Hiilesmaa, V., Paavonen, J. \& Ylikorkala, O. (2006) Clinical presentation and risk factors of placental abruption. Acta Obstetricia et Gynecologica Scandinavica 85, 700-705.

Tikkanen., M. (2010) Etiology, clinical manifestations, and prediction of placental abruption,." Acta Obstetricia et Gynecologica Scandinavica 89, 732-740.

Wandabwa, J., Doyle, P., Paul, K., Wandabwa, M.A., \& Aziga, F. (2007) Risk factors for severe abruptio placenta in Mulago Hospital, Kampala, Uganda. African Health Sciences 5, 285290.

Witlin, A.G. \& Sibai, B.M. (2001) Perinatal and maternal outcome following abruptio placentae. Hypertension in Pregnancy 20, 195-203.

Yamane, T. (1967) Statistics: an Introductory Analysis. New York: Harper \& Row. 